\title{
Immersive Rear Projection on Curved Screens
}

\author{
Andreas Kolb* $\quad$ Martin Lambers ${ }^{\dagger} \quad$ Severin Todt ${ }^{\ddagger} \quad$ Nicolas Cuntz ${ }^{\S} \quad$ Christof Rezk-Salama $^{\mathbb{I l}}$ \\ Computer Graphics Group, Institute for Vision and Graphics, University of Siegen, Germany
}

\begin{abstract}
We present a new VR installation at the University of Siegen, Germany. It consists of a $180^{\circ}$ cylindrical rear-projection screen and a front-projection floor, allowing both immersive VR applications with user tracking and convincing presentations for a larger audience.

Index Terms: I.3.7 [Computer Graphics]: Three-Dimensional Graphics and Realism-Virtual Reality

\section{INTRODUCTION}

Most existing VR installations focus either on immersive applications for a small user group including tracking and sophisticated user interaction, or on theater-like presentations for larger groups without tracking and without or with simplified user interaction.

The Computer Graphics Group at the University of Siegen recently installed a Virtual Reality laboratory for research and teaching purposes. This laboratory has unique properties, most prominently a cylindrical rear-projection screen in combination with user tracking. This allows both immersive VR applications and convincing presentations for a larger audience. In the following, we explain the design decisions that were made, identify the associated challenges, describe the implemented solutions, and discuss ideas for future improvements.
\end{abstract}

\section{HARDWARE}

We use a cylindrical curved screen with four rear-projection stereo channels and a floor with two front-projection stereo channels. Each channel has a resolution of $1400 \times 1050$ pixels. The radius of the screen is $2.5 \mathrm{~m}$ and its height is $2.6 \mathrm{~m}$. The opening angle of the cylinder is nearly $180^{\circ}$. See Fig. 1 and 2 . Infitec ${ }^{\mathrm{TM}}$ is used for stereo separation.

Tracking is done with an infrared optical tracking system from A.R.T. [1] with four cameras located at the top rim of the cylindrical screen. The tracking system provides tracked glasses, a flystick, body tracking targets, and support for custom tracking targets.

The rendering hardware consists of a Linux-based PC cluster with six render nodes and one master node. Each render node is equipped with a dual core CPU and two commodity graphics cards commonly used for high-end gaming systems. This allows to use one CPU core and one graphics card for each of the left and right channels in one stereo channel. The master node is used for synchronization and management of the cluster. Additional nodes manage the tracking system and sound. The cluster is connected using gigabit Ethernet.

The video signals generated by the render nodes are sent to Christie CineIPM [2] video processors before being passed to the

\footnotetext{
*e-mail: andreas.kolb@uni-siegen.de

†e-mail: martin.lambers@uni-siegen.de

†e-mail: severin.todt@uni-siegen.de

$\S$ e-mail: nicolas.cuntz@uni-siegen.de

Ile-mail: christof.rezk@uni-siegen.de
}

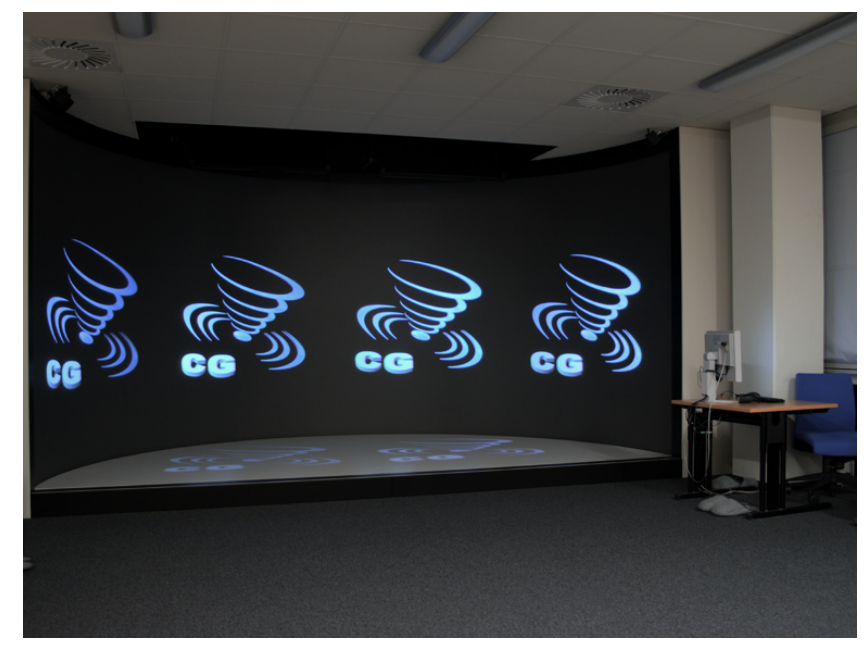

Figure 1: The VR laboratory at the University of Siegen.

projectors. These video processors perform blending for the four overlap areas (see Fig. 2), masking for the non-rectangular floor, and static warping for the curved screen (see section 3 for details). Additionally, they are used for color and brightness correction.

\section{Two-PASS WARPING}

A curved screen requires the computer-generated images to be warped before being projected onto the screen [4]. With fixed parameters, this warping is only correct for a single, ideal user position. This is good enough for presentations for larger groups of people, since here the viewers are relatively near the optimal viewer position. A tracked user that moves inside the half cylinder, however, will experience intolerable geometric distortions if the warping is not adapted to his current position (see Fig. 3).

In contrast to [4], we do not perform the complete warping dynamically in the VR application. Instead, we split the warping into a static and a dynamic pass.

The static warping pass is performed by the CineIPM video processors for the fixed sweet spot (in our case the center of the system). This has two advantages. First, since the CineIPMs use standard free-form deformation instead of quadric transfers, this allows to correct setup-specific distortions such as geometric inaccuracies. Second, applications that target presentations for larger groups need not care about warping issues.

The dynamic warping pass is performed on the graphics processing unit (GPU) by applications that support head tracking. The current head position is analytically reprojected onto the sweet spot. The application renders the scene into a texture and uses a fragment shader to warp it. The warping is completed by the static warping pass performed by the video processors.

\section{Sample applications}

In the following we describe two current research projects that have been integrated into our VR system within a few weeks only. 


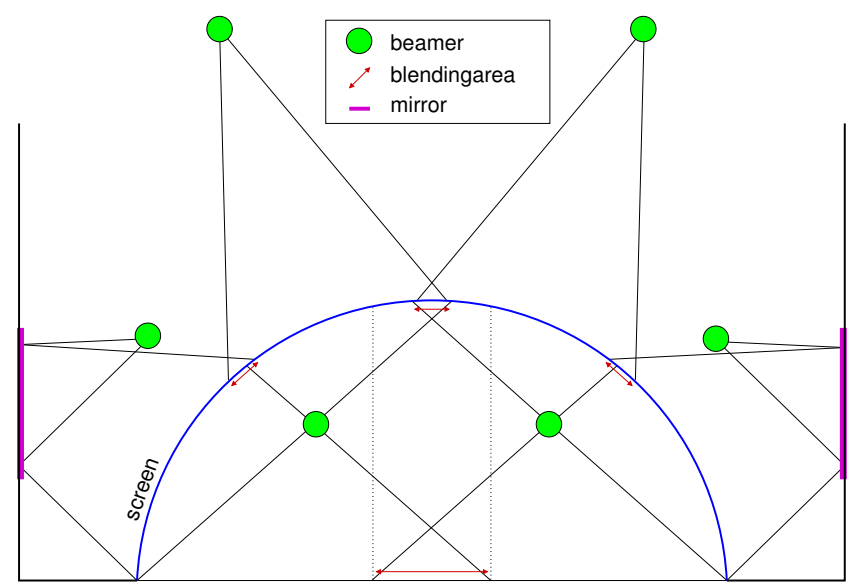

Figure 2: Plan of the VR laboratory (view from above).
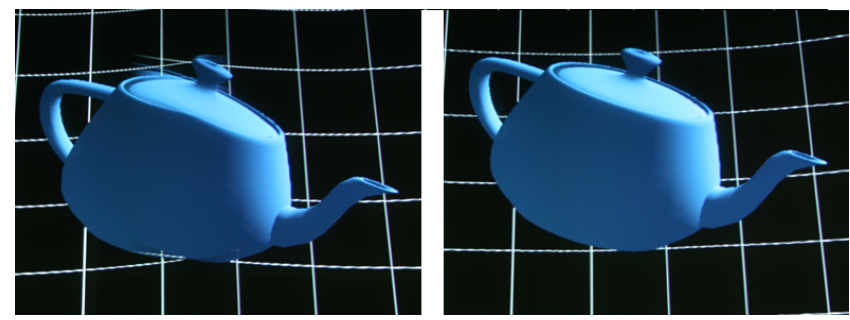

Figure 3: View of a tracked user not located near the sweet spot. In the left image, only the static warping pass is enabled. In the right image, both the static and the dynamic warping pass are enabled.

\subsection{Hase3d: Interactive Flow Visualization}

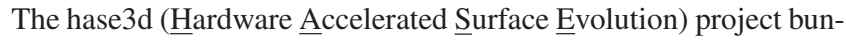
dles a set of techniques for the interactive evolution and visualization of various flow primitives, i.e. particles, lines, and surfaces/volumes. The program has been extended to multi-node rendering using the Equalizer framework.

The main application (see Fig. 4) consists of a climate visualization (originating from the approach presented by Cuntz et al. [3]), in which the user can introduce particles into atmospheric flow. Direct visualization techniques can help climate researchers to explore flow characteristics by controlling the visual output. The flow represents a pre-simulated typhoon provided by the DKRZ (German Climate Computing Centre) Hamburg.

\subsection{Volume Visualization}

Like flow visualization, 3D visualization of tomographic scans (CT, MRI) significantly benefits from virtual environments for applications both in medicine and material science. Our framework accommodates a state-of-the-art GPU-based raycaster which also utilizes the Equalizer API. Although stereo projection already improves the perception of spatial structures significantly, the immersion thrives on advanced illumination techniques. We add global illumination and scattering effects implemented using a parallel Monte-Carlo raycasting technique [5]. For real-time performance, the number of rays per pixel need to be slightly reduced (compared to a production quality). Although this will introduce a slight level of noise into the VR images, the benefits for perception are considerable. For stochastic sampling techniques, however, it is important to synchronize the pseudo random number generation among the rendering clients to create consistent images for both eyes and tiled rendering. Fig. 5 shows a non-polygonal isosurface from a

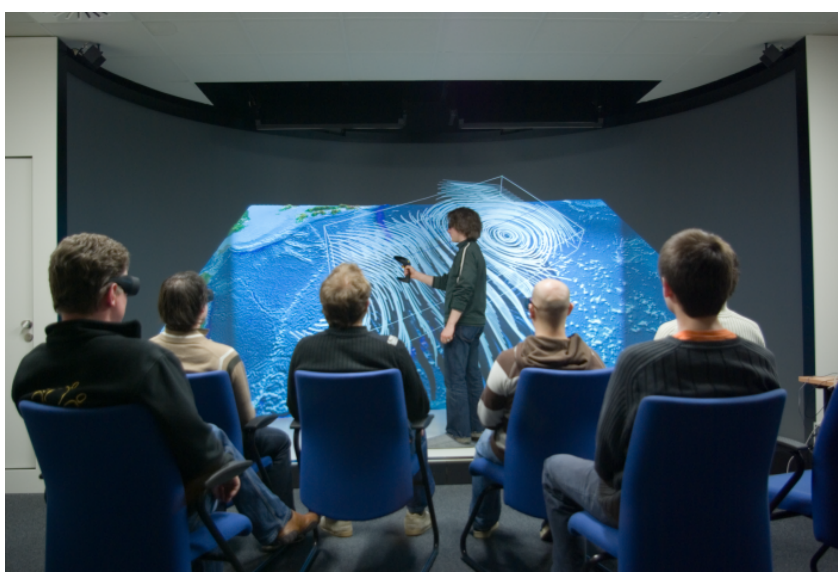

Figure 4: Hase3d: Interactive presentation for a larger group

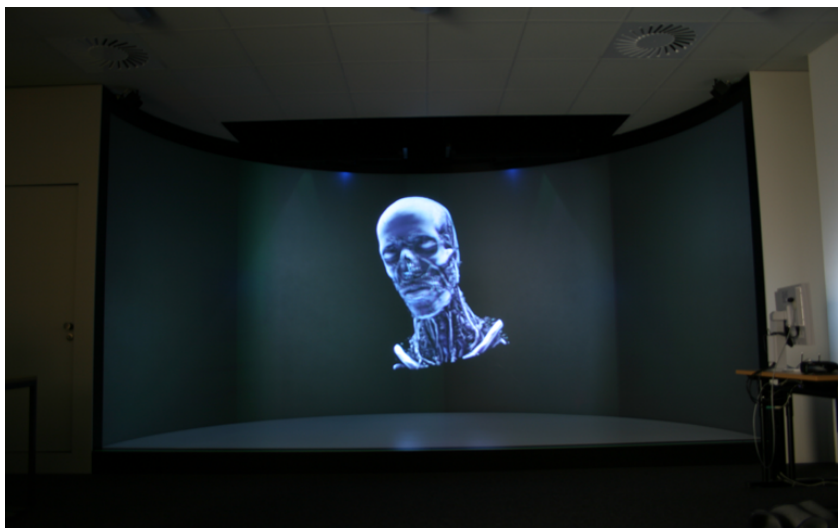

Figure 5: Our immersive volume raycasting system uses advanced illumination techniques, such as ambient occlusion, to optimize the perception of spatial relationships.

CT angiography data set rendered with ambient occlusion in realtime.

\section{CONCLUSION}

Rear projection onto curved screens offers the possibility to build flexible VR installations that can be used both for immersive application with user tracking and for interactive presentations for larger audiences. The Computer Graphics Group at the University of Siegen successfully employs a VR laboratory that makes use of these advantages.

\section{REFERENCES}

[1] Advanced Realtime Tracking GmbH. http://www.ar-tracking.de/.

[2] Christie Digital Systems. Cine-IPM 2K: Digital cinema image processing module. http://www.christiedigital.com/.

[3] N. Cuntz, A. Kolb, M. Leidl, C. Rezk-Salama, and M. Böttinger. GPUbased dynamic flow visualization for climate research applications. In Proc. SimVis, pages 371-384, 2007.

[4] R. Raskar, J. van Baar, T. Willwacher, and S. Rao. Quadric transfer for immersive curved screen displays. Comput. Graph. Forum, 23(3):451460, 2004.

[5] C. Rezk-Salama. GPU-Based Monte-Carlo Volume Raycasting. In Proc. Pacific Graphics, 2007. 